\title{
Running speed does not influence the asymmetry of kinematic variables of the lower limb joints in novice runners
}

\author{
XINYAN JIANG ${ }^{1}$, HAIRONG CHEN ${ }^{1}$, DONG SUN ${ }^{1}$, JULIEN S. BAKER ${ }^{2}$, YAODONG GU $^{1 *}$ \\ ${ }^{1}$ Faculty of Sports Science, Ningbo University, Ningbo, China. \\ ${ }^{2}$ Department of Sport, Physical Education and Health, Hong Kong Baptist University, Hong Kong, China.
}

\begin{abstract}
Purpose: The purpose of this study was to evaluate kinematic variables at 5 running speeds ( 8 to $12 \mathrm{~km} / \mathrm{h}$ ), for the asymmetry between lower limbs. We also investigated the effects of running speed on the bilateral asymmetry of the lower limb joints kinematic variables among novice runners. Methods: Kinematic $(200 \mathrm{~Hz})$ running data were collected bilaterally for 17 healthy male novice runners (age: $23.1 \pm 1.3$ years, height: $1.77 \pm 0.04 \mathrm{~m}$, mass: $72.3 \pm 4.57 \mathrm{~kg}$, BMI: $23.1 \pm 1.0 \mathrm{~kg} / \mathrm{m}^{2}$ ) running on a treadmill at 5 fixed speeds $(8,9,10,11$ and $12 \mathrm{~km} / \mathrm{h})$ in a randomized order. Symmetry angles (SA) were calculated to quantify gait asymmetry magnitude at each running speed. Results: Overall, SPM analysis using paired $t$-tests revealed significant joints kinematic differences between the left lower limb and the right lower limb at each running speed. Significant differences between limbs were found for all joint kinematic variables in the ankle and hip, regardless of running speed. As for the knee angle, significant differences between legs were only found during the running speed of $11 \mathrm{~km} / \mathrm{h}$. For knee angle velocity, significant differences between legs were found in all running speeds except for $8 \mathrm{~km} / \mathrm{h}$. However, there was no noticeable difference in asymmetry values across running speeds. Conclusions: The findings of the current study indicate that gait asymmetry of joint kinematics variables between lower limbs during running is apparent in healthy novice runners. Meanwhile, running speed does not influence lower limb joints kinematic asymmetry among novice runners.
\end{abstract}

Key words: novice runner, symmetry, running speed, kinematics

\section{Introduction}

Recreational running is a popular form of physical exercise. Although running has a positive effect on physical health and well-being, it also increases the occurrence of sustaining a running-related injury (RRI) [7]. The occurrence of RRI has been reported at $79 \%$, which is contradictory to the positive health effects [18]. The number of RRI being particularly high in novice runners [6]. The literature [7] has outlined that variables such as higher BMI, increased age, previous injuries and previous sports activity participation are related to RRI in novice runners. However, there is little consistency in studies regarding the specific causes of RRI.
It is generally believed that suboptimal lower limb movement patterns may increase injury risk in runners. Bilateral asymmetry between the lower limbs during running has been considered a risk factor [29]. Since many running-related injuries are unilateral, asymmetrical running may be an overlooked cause of injury as related compensatory actions could put excessive loads on the body [28]. Alternatively, asymmetries may be an early indicator of potential injury [21]. The completely symmetrical gait of running is not possible because of the dominant leg. Lower extremity dominance has been classified based on the leg's role in either stabilization or mobilization. For example, the leg used for kicking a ball is considered as the mobilization leg and the stance leg is the stabi-

\footnotetext{
* Corresponding author: Yaodong Gu, Ningbo University, No. 818 Fenghua Road, Ningbo, 315211, China. Phone: 8657487609369, e-mail: guyaodong@hotmail.com

Received: October 25th, 2020

Accepted for publication: January 5th, 2021
} 
lization leg [24]. The symmetry of gait, the perfect coordination between the right leg and left leg measures, may not occur in healthy populations [26]. Subtle asymmetry is not detectable by the clinician's vision, nor is it necessarily kinaesthetically apparent to the athlete. Therefore, a biomechanical analysis of running asymmetry is needed.

Biomechanical asymmetry is not detrimental during walking tasks, however, it could potentially become pathological or injurious during running due to the increased biomechanical demands placed on the musculoskeletal system [24]. Asymmetrical gait patterns during running have been reported widely in previous studies. Bredeweg et al. [5] found that, compared to uninjured novice runners, the injured novice runners showed lower bilateral asymmetry in peak impact force and contact time. Robadey et al. [25] found that the injured side exhibited significantly smaller values for biomechanical parameters, compared to the uninjured leg during overground running. Radzak et al. [24] concluded that many of the biomechanical variables (e.g., loading rate and stiffness) previously associated with overuse injuries of the lower limbs were found to be asymmetrical in the rested state and remained asymmetrical in the fatigued state. Gao et al. [13] found that during running stance, the symmetry of the hip flexion angle, knee flexion angle, hip extension angle and hip flexion moment was decreased due to fatigue from prolonged running, and the knee extension velocity and hip flexion velocity increased. The alterations of asymmetry can be considered as a compensation mechanism to maintain gait stability during running.

During running, the lower extremities act as a pair of oscillators. Theoretically, as the speed increases, running gait becomes more symmetrical due to the perfect phrasing of gait to achieve a faster speed [19]. However, studies of biomechanical variability in different running speeds are usually applied to unilateral limbs [11], [17]. Only a few studies have investigated bilateral asymmetry in the lower extremity during running with different speeds, however, findings of previous studies were inconsistent. Bredeweg et al. [5] found that male recreational runners presented no significant differences in bilateral asymmetry during running at the speed of 9 and $10 \mathrm{~km} / \mathrm{h}$, while female recreational runners showed significantly smaller bilateral asymmetry at $9 \mathrm{~km} / \mathrm{h}$, compared to $8 \mathrm{~km} / \mathrm{h}$. Furlong et al. [12] reported smaller bilateral asymmetry during running at preferred speed than nonpreferred speed in healthy young adult males. Limit test speeds were adopted in the most previous study. Mo et al. [19] reported that competitive runners ex- hibited a linear reduction in bilateral asymmetry with increasing running speed from 8 to $12 \mathrm{~km} / \mathrm{h}$, while the bilateral asymmetry of recreational runners exhibited a roughly U-shaped trend across speeds, as for novice runners, changes of bilateral asymmetry across speed were inconsistent. Girard et al. [15] concluded running speed does not change lower limb mechanical asymmetry by examining the magnitude and range of asymmetry at seven running velocities $(10,12.5,15$, $17.5,20,22.5$, and $25 \mathrm{~km} / \mathrm{h}$ ). However, the aforementioned studies [15], [19] focused on the kinetics of bilateral asymmetry during running at different speeds. Thus, it remains inconclusive how the bilateral asymmetry of lower limb joints kinematics changes across running speeds.

The main purpose of this study was to investigate the effect of running speed on the bilateral asymmetry of lower limb joints kinematic variables of novice runners. We also determined differences in running kinematics of the left leg and right leg between running speed from 8 to $12 \mathrm{~km} / \mathrm{h}$ using statistical parametric mapping (SPM). We hypothesized that there would be differences between running speed and bilateral asymmetry of lower limb joints kinematics. We also hypothesized that the left leg and right leg exhibit different joint kinematics in the stance phase of running by analysing the results of statistical parametric mapping.

\section{Methods}

\subsection{Participants}

Seventeen healthy male novice runners (age: 23.1 \pm 1.3 years, height: $1.77 \pm 0.04 \mathrm{~m}$, mass: $72.25 \pm 4.57$ $\mathrm{kg}$, BMI: $23.1 \pm 1.0 \mathrm{~kg} / \mathrm{m}^{2}$ ) from Ningbo University were recruited as experimental subjects in this study. All participants were free from health problems and/ or neuromuscular disorders and/or known gait impairments, and had no lower limb injuries in the previous six months. The dominant leg was defined as the preferred leg when kicking a ball and participants' right-side limb was the dominant limb. All participants were rearfoot striking runners. Runners who had never participated in any running competitions or performed regular running exercises (i.e., at least 3 times per week, 30 minutes per time, and minimum weekly running distance of $20 \mathrm{~km}$ ) for less than 24 months were defined as novice runners [1]. Before the experiment, all participants were provided 
with the documented consent approved by the Institutional Review Board.

\subsection{Experimental procedures}

All participants were instructed to run on a motorized treadmill (Satun h/p/cosmos, Nussdorf- Traunstein, Nußdorf, Germany) at five fixed speeds $(8,9$, 10,11 and $12 \mathrm{~km} / \mathrm{h}$ ) with the slope of the treadmill set at $0^{\circ}$. Running speeds were assigned in a randomized order among participants. Before testing, participants had 10 minutes warm-up and to familiarize themselves with experimental settings. During running, participants were required to run for $3 \mathrm{~min}$ at each running speed and kinematics data were recorded. All participants were asked to use their own running shoes and were allowed to have a rest for 5 minutes between each run. An eight-camera motion capture system (Vicon Metrics Ltd., Oxford, United Kingdom) was used to record running kinematic data at a frequency of $200 \mathrm{~Hz} .36$ retroreflective markers fixed to the lower limb of each runner to track movement, detailed in Fig. 1.
Inc., Germantown, MD, USA) was applied to process and calculate kinematic variables in the sagittal plane of the ankle, knee and hip joints using C3D files generated from Vicon Nexus Software. Kinematics data were filtered by $10 \mathrm{~Hz}$ fourth-order lowpass Butterworth filter for the de-noising process of marker trajectories [23]. The joint angles were calculated by the inverse kinematics algorithm in Visual 3D. The kinematic data were processed using MATLAB R2019a (The MathWorks, MA, United States), and the written script was applied to process the data.

The symmetry angle (SA) was used to evaluate the level of lower limb bilateral asymmetry as in previous studies [13], [15], [24]. In the current study, for each participant, SA was calculated for inter-leg symmetry and rectified so that all symmetry values were positive. The SA was calculated using the following formula [30]:

$$
S A=\frac{\left(45^{\circ}-\arctan \left(X_{\text {left }} / X_{\text {right }}\right)\right)}{90^{\circ}} \times 100 \%
$$

but if $\left(45^{\circ}-\arctan \left(X_{\text {left }} / X_{\text {right }}\right)\right)>90^{\circ}$.
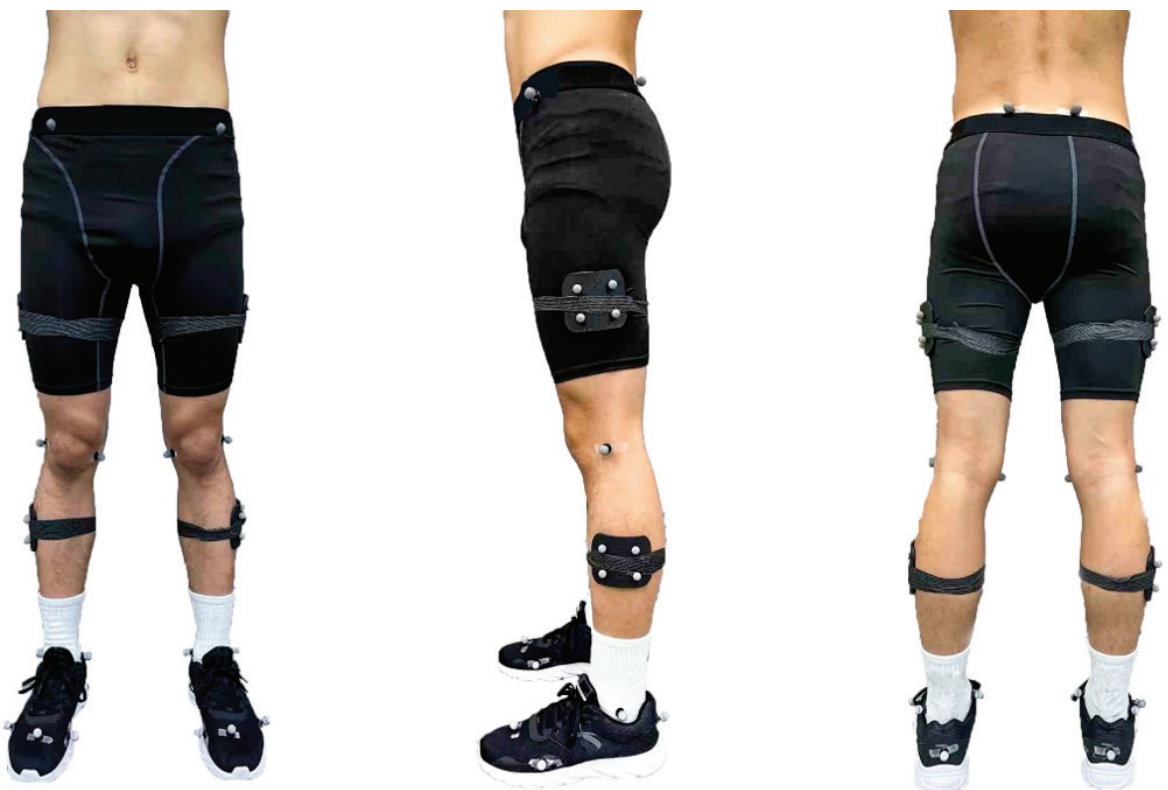

Fig. 1. Illustration of retroreflective marker placement

\subsection{Data processing}

This study focused on the changes in the sagittal plane for specific interest, as there is reported primary variation in the sagittal plane during running [3]. A customized function in Visual 3D (c-motion
Then

$$
S A=\frac{\left(45^{\circ}-\arctan \left(X_{\text {left }} / X_{\text {right }}\right)-180^{\circ}\right)}{90^{\circ}} \times 100 \%,
$$

$S A$ was presented as a percentage, $X$ represents the value of the kinematic variables in the right or left 
lower limb. A score of $0 \%$ suggests perfect symmetry and $100 \%$ suggests perfect asymmetry between the right and left leg [15].

\subsection{Statistical analysis}

Ten consecutive steps (5 right and 5 left foot contacts) beginning at the second minute of each three minutes running bout were analysed. Descriptive statistics were provided as means and standard deviations (SD). Tests for normality and homogeneity of variances (Shapiro-Wilk and Levene's, respectively) were conducted on all SA data before the analysis. Repeated measures ANOVA was performed to determine the effects of running speed on bilateral asymmetry during running, and Tukey's post hoc tests were used to analyse the specific significant differences. Paired $t$-tests assessed differences in kinematic variables between legs at different running speeds. All statistical calculations were carried out with SPSS 25.0 for Windows ${ }^{\mathrm{TM}}$ software (IBM, Armonk, NY, USA). The level of statistical significance was set at $p<0.05$.

For statistical parametric mapping (SPM), kinematic data for each step were time normalized to the stance phase (101 data points per stance phase). Biomechanical differences between the right and left lower limb of each running speed were obtained by statistically examining the entire times series using SPM with post hoc paired $t$-tests. The significance level for all statistical significance was set at $p<0.05$. All SPM analyses were conducted in MATLAB R2019a (The MathWorks, MA, United States) using the open-source software package spm1D 0.4 [22] (www.spm1d.org).

\section{Results}

Significant differences between the lower limbs were analysed via $t$-tests at different running speed states, indicating limb asymmetry, are exhibited in Table 1 and Figs. 2-6, respectively. At the $8 \mathrm{~km} / \mathrm{h}$ condition, significant differences between the legs were found in the peak ankle dorsiflexion angle $(p=0.007)$, peak ankle plantarflexion angle $(p=0.001)$ and peak hip flexion angle $(p=0.006)$. At the $9 \mathrm{~km} / \mathrm{h}$ condition, significant differences between the limbs were found in peak ankle plantarflexion angle $(p<0.001)$, peak ankle plantarflexion velocity $(p<0.001)$, peak knee flexion angle $(p=0.029)$ and peak hip flexion angle $(p=$ 0.003 ). At the running speed of $10 \mathrm{~km} / \mathrm{h}$, significant differences between the limbs were found in peak ankle plantarflexion angle $(p=0.003)$, peak ankle plantarflexion velocity $(p=0.045)$ and peak knee flexion velocity $(p=0.001)$. At $11 \mathrm{~km} / \mathrm{h}$ condition,

Table 1. Comparison of selected kinematic variables between right and left limbs at 5 fixed running speeds

\begin{tabular}{|c|c|c|c|c|c|}
\hline \multicolumn{3}{|c|}{ Joint Kinematics } & \multirow{2}{*}{$\begin{array}{c}\text { Left Limb (SD) } \\
\mathbf{4}\end{array}$} & \multirow{2}{*}{$\begin{array}{c}\text { Right Limb (SD) } \\
\mathbf{5}\end{array}$} & \multirow{2}{*}{$\frac{p \text {-value }}{6}$} \\
\hline $\mathbf{1}$ & 2 & 3 & & & \\
\hline \multirow{16}{*}{ Ankle } & \multirow{4}{*}{$8 \mathrm{~km} / \mathrm{h}$} & Dorsiflexion $\left[{ }^{\circ}\right]$ & $18.18(1.31)$ & $14.74(4.51)$ & 0.007 \\
\hline & & Plantarflexion $\left[{ }^{\circ}\right]$ & $25.57(4.97)$ & $21.02(3.71)$ & 0.001 \\
\hline & & Dorsiflexion velocity [ $\%$ s] & $184.60(30.81)$ & $177.78(29.04)$ & 0.468 \\
\hline & & Plantarflexion velocity [\%/s] & $342.63(50.83)$ & $351.39(39.13)$ & 0.473 \\
\hline & \multirow{4}{*}{$9 \mathrm{~km} / \mathrm{h}$} & Dorsiflexion $\left[{ }^{\circ}\right]$ & $16.82(3.22)$ & $14.54(5.76)$ & 0.217 \\
\hline & & Plantarflexion $\left[{ }^{\circ}\right]$ & $25.73(4.16)$ & $20.17(3.21)$ & 0.000 \\
\hline & & Dorsiflexion velocity $[\%$ s] & $183.07(24.94)$ & $187.44(24.50)$ & 0.579 \\
\hline & & Plantarflexion velocity [\%/s] & $352.04(36.80)$ & $415.62(50.01)$ & 0.000 \\
\hline & \multirow{4}{*}{$10 \mathrm{~km} / \mathrm{h}$} & Dorsiflexion $\left[{ }^{\circ}\right]$ & $16.70(3.93)$ & $15.77(5.48)$ & 0.480 \\
\hline & & Plantarflexion $\left[{ }^{\circ}\right]$ & $25.49(4.09)$ & $22.60(3.96)$ & 0.003 \\
\hline & & Dorsiflexion velocity [\%] & $188.30(19.01)$ & $196.00(26.62)$ & 0.465 \\
\hline & & Plantarflexion velocity $[\% / \mathrm{s}])$ & $420.50(29.77)$ & $455.43(56.40)$ & 0.045 \\
\hline & \multirow{4}{*}{$11 \mathrm{~km} / \mathrm{h}$} & Dorsiflexion $\left[{ }^{\circ}\right]$ & $18.10(2.69)$ & $15.89(5.28)$ & 0.150 \\
\hline & & Plantarflexion $\left[{ }^{\circ}\right]$ & $27.06(3.75)$ & $21.38(3.71)$ & 0.000 \\
\hline & & Dorsiflexion velocity [\%] & $202.24(14.78)$ & $191.70(25.70)$ & 0.229 \\
\hline & & Plantarflexion velocity [\%/s] & $428.09(34.93)$ & $448.18(58.64)$ & 0.292 \\
\hline
\end{tabular}




\begin{tabular}{|c|c|c|c|c|c|}
\hline 1 & 2 & 3 & 4 & 5 & 6 \\
\hline \multirow{4}{*}{ Ankle } & \multirow{4}{*}{$12 \mathrm{~km} / \mathrm{h}$} & Dorsiflexion $\left[{ }^{\circ}\right]$ & $19.42(1.25)$ & $14.87(4.95)$ & 0.001 \\
\hline & & Plantarflexion $\left[^{\circ}\right]$ & $27.95(6.13)$ & $20.84(3.13)$ & 0.000 \\
\hline & & Dorsiflexion velocity [\% $/ \mathrm{s}]$ & $194.03(25.14)$ & $195.82(30.32)$ & 0.847 \\
\hline & & Plantarflexion velocity $[\% / \mathrm{s}]$ & $458.18(24.83)$ & $468.41(57.73)$ & 0.463 \\
\hline \multirow{20}{*}{ Knee } & \multirow{4}{*}{$8 \mathrm{~km} / \mathrm{h}$} & Flexion $\left[{ }^{\circ}\right]$ & $32.14(5.40)$ & $32.33(5.07)$ & 0.928 \\
\hline & & Extension $\left[{ }^{\circ}\right]$ & $-9.84(3.68)$ & $-7.15(6.34)$ & 0.181 \\
\hline & & Flexion velocity $[\% / \mathrm{s}]$ & $291.91(47.32)$ & $289.04(29.76)$ & 0.823 \\
\hline & & Extension velocity $[\% / \mathrm{s}]$ & $160.16(32.00)$ & $149.86(36.12)$ & 0.150 \\
\hline & \multirow{4}{*}{$9 \mathrm{~km} / \mathrm{h}$} & Flexion $\left[{ }^{\circ}\right]$ & $30.36(5.16)$ & $34.25(5.12)$ & 0.029 \\
\hline & & Extension $\left[{ }^{\circ}\right]$ & $-9.24(2.72)$ & $-6.30(5.95)$ & 0.092 \\
\hline & & Flexion velocity $[\%]$ & $345.46(75.86)$ & $309.59(29.64)$ & 0.116 \\
\hline & & Extension velocity $[\% / \mathrm{s}]$ & $199.44(51.27)$ & $165.02(30.46)$ & 0.051 \\
\hline & \multirow{4}{*}{$10 \mathrm{~km} / \mathrm{h}$} & Flexion $\left[^{\circ}\right]$ & $32.65(6.22)$ & $35.29(5.97)$ & 0.255 \\
\hline & & Extension $\left[{ }^{\circ}\right]$ & $-11.36(5.30)$ & $-7.73(8.09)$ & 0.122 \\
\hline & & Flexion velocity $[\%]$ & $332.44(38.30)$ & $297.38(21.91)$ & 0.001 \\
\hline & & Extension velocity $[\% / \mathrm{s}]$ & $179.83(40.10)$ & $184.97(25.98)$ & 0.471 \\
\hline & \multirow{4}{*}{$11 \mathrm{~km} / \mathrm{h}$} & Flexion $\left[{ }^{\circ}\right]$ & $32.63(5.06)$ & $36.52(3.93)$ & 0.065 \\
\hline & & Extension $\left[{ }^{\circ}\right]$ & $-10.87(3.91)$ & $-6.89(8.56)$ & 0.137 \\
\hline & & Flexion velocity $[\% / \mathrm{s}]$ & $341.13(26.80)$ & $303.88(22.20)$ & 0.000 \\
\hline & & Extension velocity $[\% / \mathrm{s}]$ & $194.81(36.09)$ & $187.99(21.54)$ & 0.403 \\
\hline & \multirow{4}{*}{$12 \mathrm{~km} / \mathrm{h}$} & Flexion $\left[{ }^{\circ}\right]$ & $33.58(5.02)$ & $35.52(4.02)$ & 0.310 \\
\hline & & Extension $\left[{ }^{\circ}\right]$ & $-11.71(3.45)$ & $-8.47(6.54)$ & 0.097 \\
\hline & & Flexion velocity $[\% / \mathrm{s}]$ & $325.34(29.62)$ & $325.41(27.65)$ & 0.994 \\
\hline & & Extension velocity $[\% / \mathrm{s}]$ & $191.11(40.82)$ & $198.94(32.19)$ & 0.422 \\
\hline \multirow{20}{*}{ Hip } & \multirow{4}{*}{$8 \mathrm{~km} / \mathrm{h}$} & Flexion $\left[{ }^{\circ}\right]$ & $23.59(2.84)$ & 26.92(3.09) & 0.006 \\
\hline & & Extension $\left[{ }^{\circ}\right]$ & $7.39(2.10)$ & $7.24(1.73)$ & 0.809 \\
\hline & & Flexion velocity [\%] & $49.67(37.75)$ & $59.59(15.55)$ & 0.345 \\
\hline & & Extension velocity $[\% / \mathrm{s}]$ & $179(30.97)$ & $181.29(17.40)$ & 0.842 \\
\hline & \multirow{4}{*}{$9 \mathrm{~km} / \mathrm{h}$} & Flexion $\left[{ }^{\circ}\right]$ & $24.70(4.45)$ & $29.54(2.31)$ & 0.003 \\
\hline & & Extension $\left[{ }^{\circ}\right]$ & $9.01(2.56)$ & $8.78(1.57)$ & 0.727 \\
\hline & & Flexion velocity $[\%]$ & $70.49(49.67)$ & $64.15(27.16)$ & 0.680 \\
\hline & & Extension velocity $[\% / \mathrm{s}]$ & $211.4(28.71)$ & $203.66(22.25)$ & 0.496 \\
\hline & \multirow{4}{*}{$10 \mathrm{~km} / \mathrm{h}$} & Flexion $\left[{ }^{\circ}\right]$ & $30.24(5.06)$ & $31.02(2.01)$ & 0.534 \\
\hline & & Extension $\left[^{\circ}\right]$ & $9.23(2.69)$ & $9.54(1.58)$ & 0.649 \\
\hline & & Flexion velocity [\%] & $60.57(32.20)$ & $56.09(25.77)$ & 0.641 \\
\hline & & Extension velocity [\%] & $242.13(44.38)$ & $240.05(26.44)$ & 0.798 \\
\hline & \multirow{4}{*}{$11 \mathrm{~km} / \mathrm{h}$} & Flexion $\left[{ }^{\circ}\right]$ & $29.29(4.26)$ & $32.78(3.07)$ & 0.012 \\
\hline & & Extension $\left[^{\circ}\right]$ & $10.38(2.53)$ & $9.87(1.54)$ & 0.393 \\
\hline & & Flexion velocity $[\%]$ & $63.34(21.87)$ & $94.60(66.99)$ & 0.126 \\
\hline & & Extension velocity $[\% / \mathrm{s}]$ & $250.69(38.65)$ & $243.47(37.21)$ & 0.401 \\
\hline & \multirow{4}{*}{$12 \mathrm{~km} / \mathrm{h}$} & Flexion $\left[{ }^{\circ}\right]$ & $29.76(5.02)$ & $33.23(3.22)$ & 0.050 \\
\hline & & Extension $\left[{ }^{\circ}\right]$ & $10.72(2.86)$ & $11.37(2.32)$ & 0.470 \\
\hline & & Flexion velocity $[\%$ s] & $52.90(14.00)$ & $85.00(54.85)$ & 0.041 \\
\hline & & Extension velocity $[\% / \mathrm{s}]$ & $274.72(50.05)$ & $251.56(25.00)$ & 0.036 \\
\hline
\end{tabular}

significant differences between the legs were found in peak ankle plantarflexion angle $(p<0.001)$ and peak knee flexion velocity $(p=0.001)$. For the running speed of $12 \mathrm{~km} / \mathrm{h}$, significant differences between the legs were found in peak ankle dorsiflexion angle $(p=0.001)$, peak ankle plantarflexion angle $(p<0.001)$, peak hip flexion velocity $(p=0.041)$ and peak hip extension velocity $(p=0.036)$. 

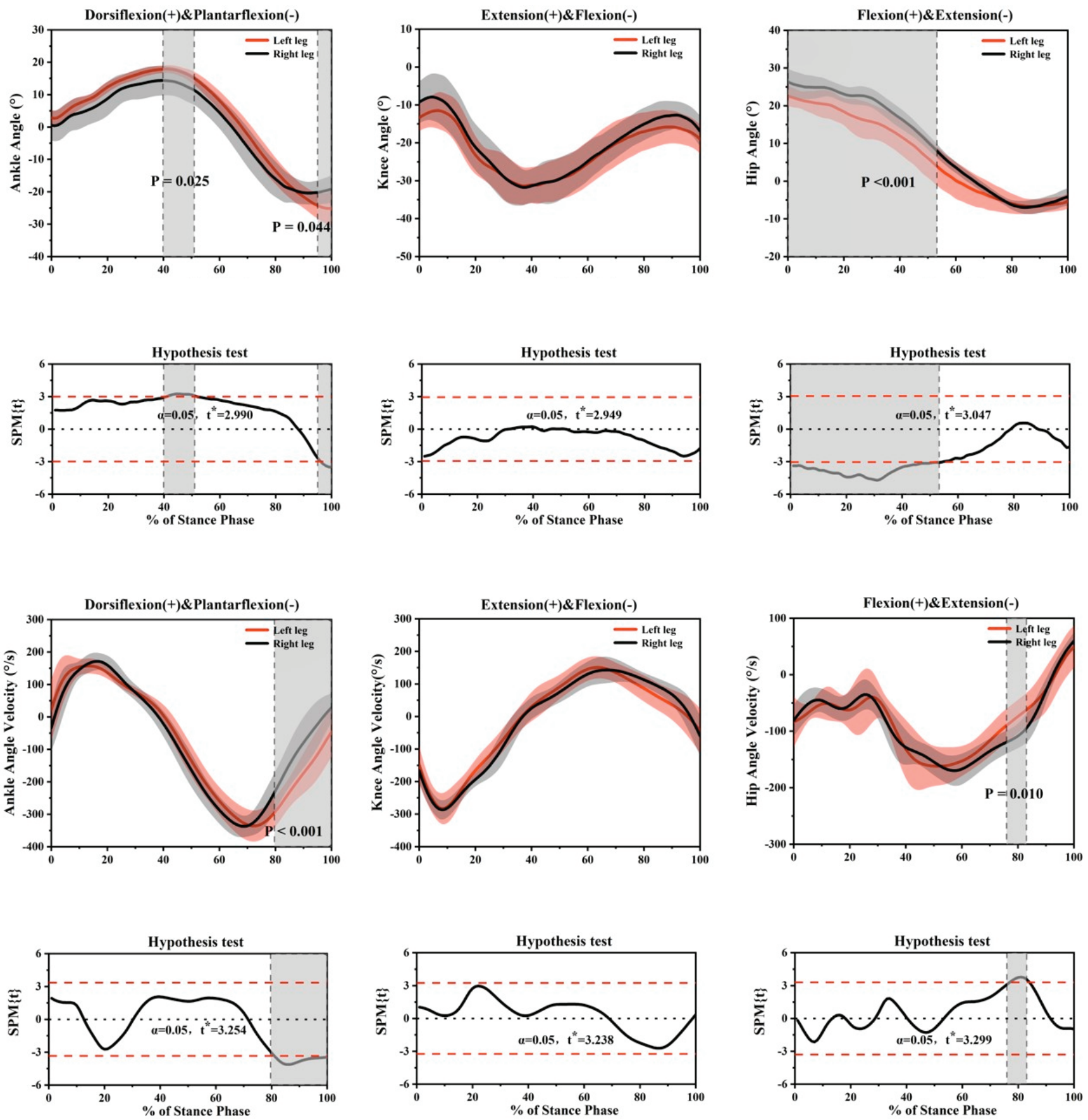

Fig. 2. Descriptive results between left lower limb and right lower limb statistical parametric mapping results during running at $8 \mathrm{~km} / \mathrm{h}, t$-values of the SPM for all participants (post hoc results; dashed red lines represent $p=0.05$ level).

Grey shaded areas present regions with statistically significant differences

The SPM analysis with paired $t$-tests revealed significant differences between the legs at different running speeds during the running stance phase. In Figures $2-6$, the joints kinematic differences of each running speed in the corresponding time series is shown. Significant differences between the limbs were found for all joint kinematic variables in ankle and hip, regardless of running speed. As for knee angle, significant differences between the legs were only found during the running speed of $11 \mathrm{~km} / \mathrm{h}$. However, for knee angle velocity, significant differences between legs were found in all running speeds except for $8 \mathrm{~km} / \mathrm{h}$. 

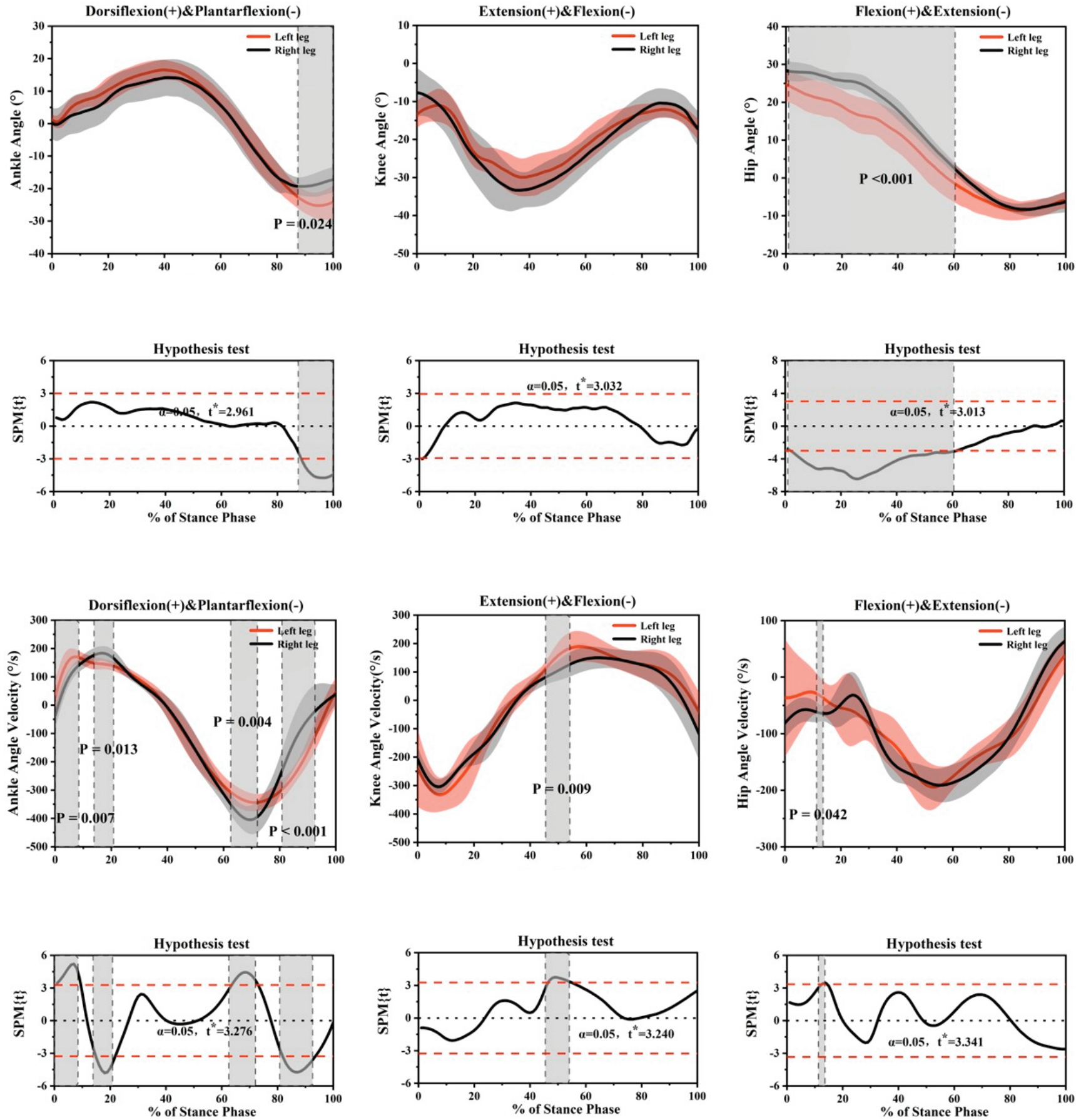

Fig. 3. Descriptive results between left lower limb and right lower limb statistical parametric mapping results during running at $9 \mathrm{~km} / \mathrm{h}, t$-values of the SPM for all participants (post hoc results; dashed red lines represent $p=0.05$ level).

Grey shaded areas present regions with statistically significant differences 

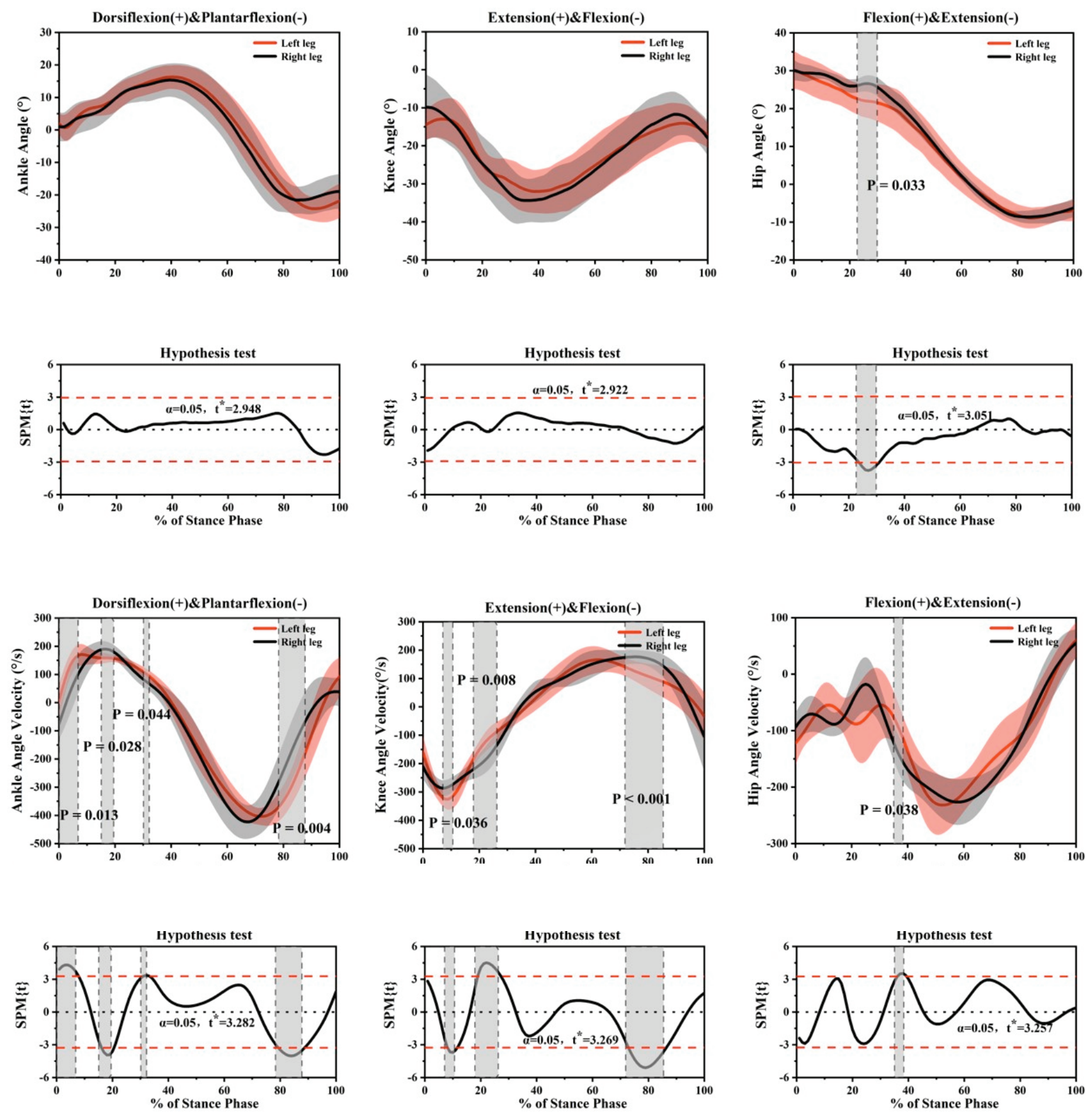

Fig. 4. Descriptive results between left lower limb and right lower limb statistical parametric mapping results during running at $10 \mathrm{~km} / \mathrm{h}, t$-values of the SPM for all participants (post hoc results; dashed red lines represent $p=0.05$ level).

Grey shaded areas present regions with statistically significant differences 

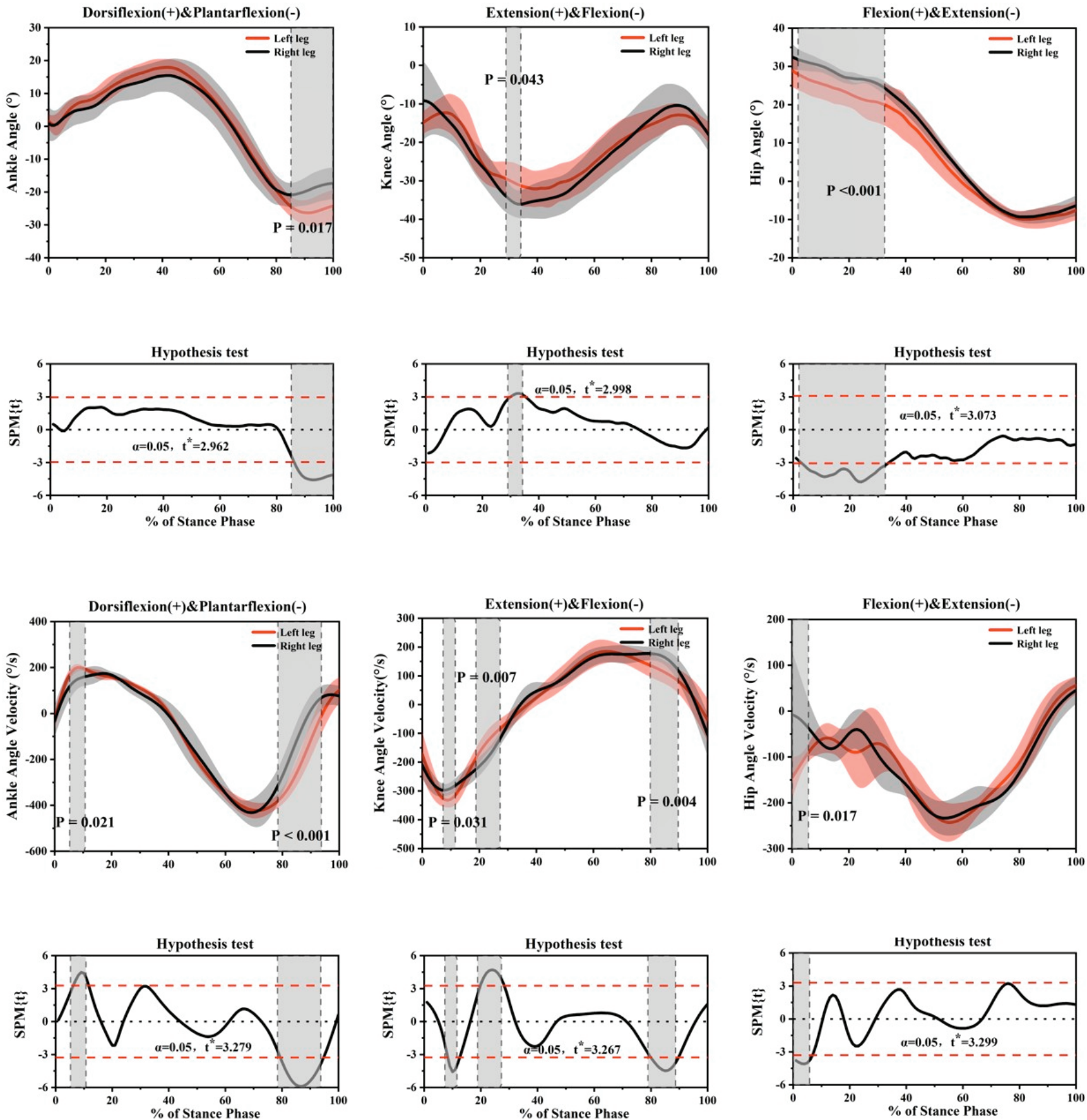

Fig. 5. Descriptive results between left lower limb and right lower limb statistical parametric mapping results during running at $11 \mathrm{~km} / \mathrm{h}, t$-values of the SPM for all participants (post hoc results; dashed red lines represent $p=0.05$ level).

Grey shaded areas present regions with statistically significant differences 

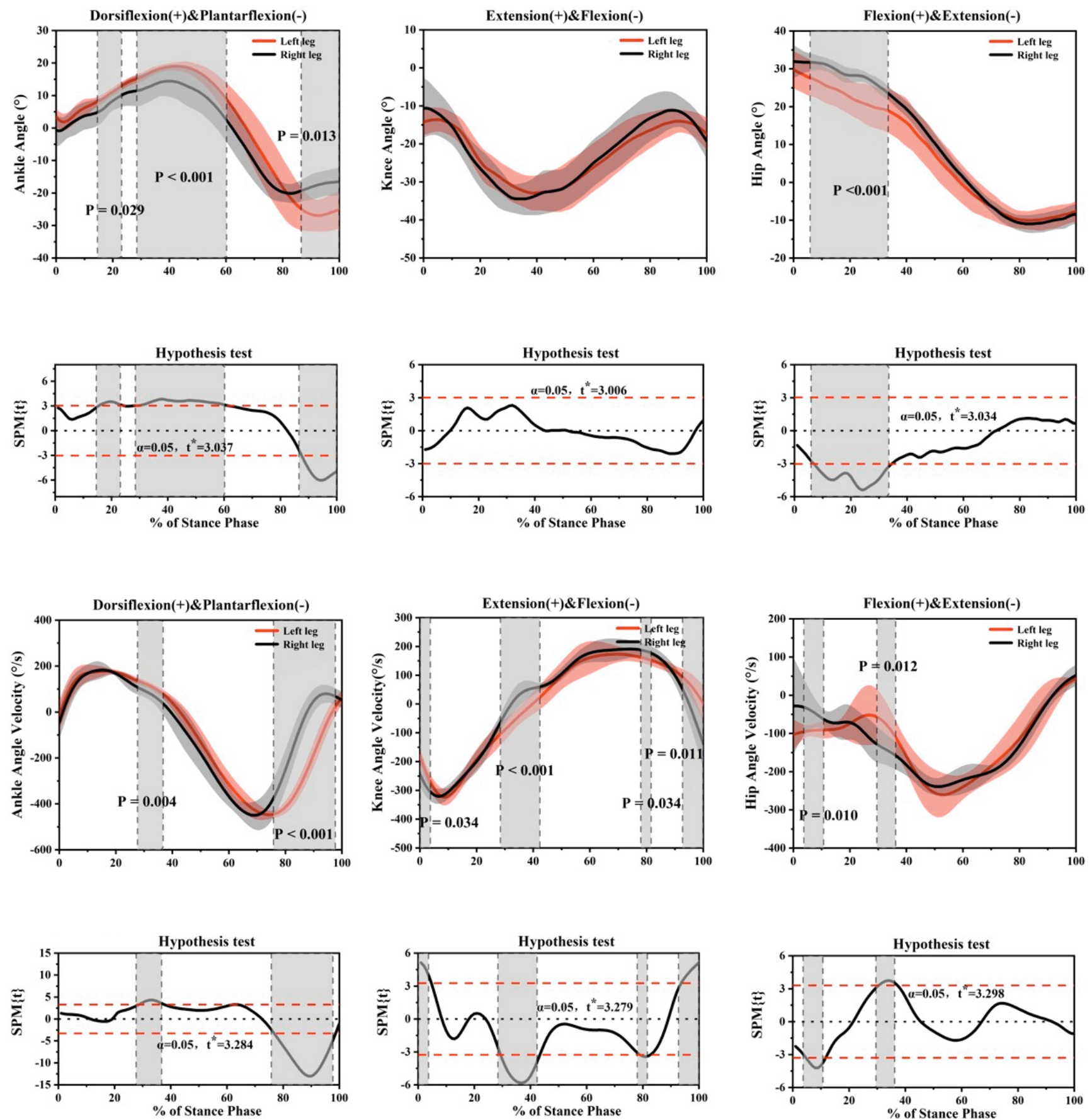

Fig. 6. Descriptive results between left lower limb and right lower limb statistical parametric mapping results during running at $12 \mathrm{~km} / \mathrm{h}, t$-values of the SPM for all participants (post hoc results; dashed red lines represent $p=0.05$ level).

Grey shaded areas present regions with statistically significant differences 
Table 2. The significance of the symmetry angle (SA) in the joint kinematic variables during different running speeds

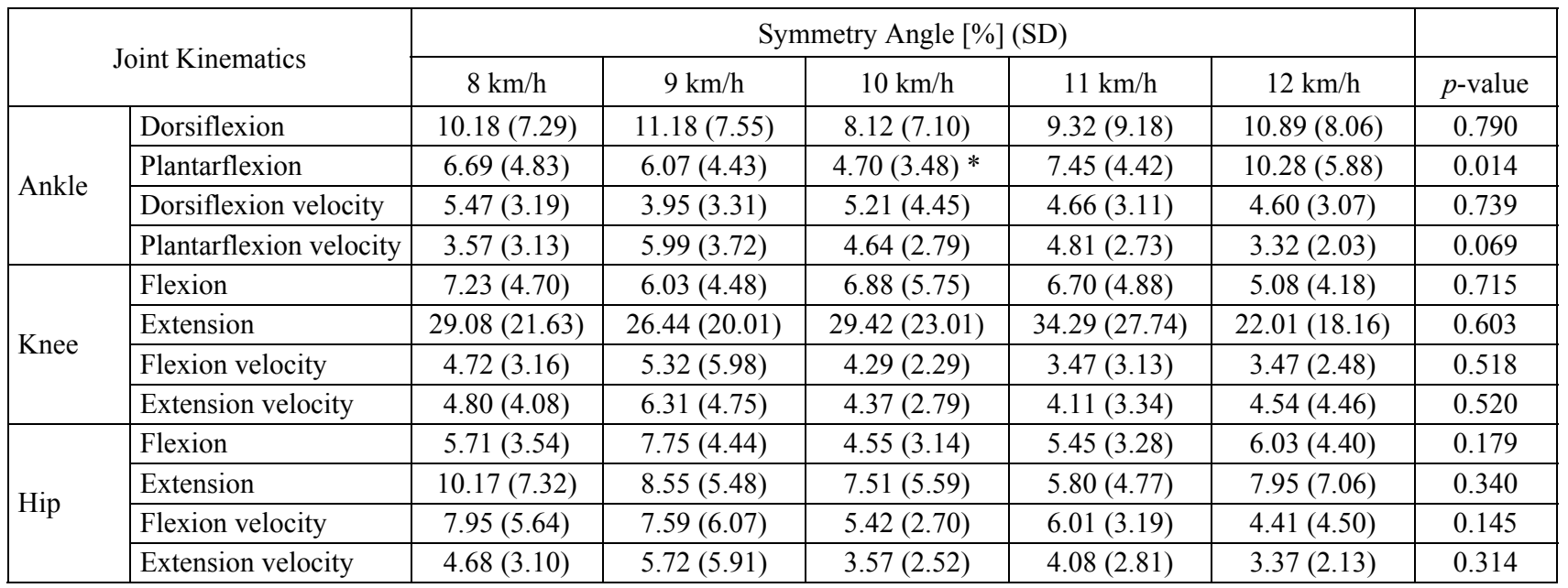

Note: “*” indicates the significance of SA in the bilateral lower limb between $10 \mathrm{~km} / \mathrm{h}$ and $12 \mathrm{~km} / \mathrm{h}(p<0.05)$.

Significant differences in SA of ankle, knee, and hip biomechanical variables between different running speed, indicating changes in lower limb asymmetry magnitude due to running speed, are presented in Table 2. Significant differences $(p=0.007)$ were only observed in the ankle plantarflexion angle between the speed conditions of 10 and $12 \mathrm{~km} / \mathrm{h}$.

\section{Discussion}

The purpose of this study was to examine kinematic bilateral asymmetry during running, with a specific interest in how bilateral asymmetry changes along with increasing running speeds among novice runners. We only investigated the biomechanical variables during the stance phase of running, as it has been reported that the running stance phase is closely associated with running-related injuries [9], [10]. Significant differences were found in kinematic measures between lower limbs in novice runners at each running speed state, which supports previous research reporting differences between lower limbs [13], [24], [27]. Runners' SA scores were analyzed at five different speeds $(8-12 \mathrm{~km} / \mathrm{h})$ to determine whether a different degree of bilateral asymmetry was present. However, there was almost no influence of running speed on bilateral asymmetry scores for any of the kinematic variables studied since our results showed relatively unchanging average values across all speeds.

The human body has a slight congenital asymmetry in form and function. The asymmetry of gait in healthy individuals was previously called functional asymmetry, that is, the asymmetry of the limbs is based on the differentiation of main functions, especially stabilization or propulsion [27]. Regardless of the speed condition, SPM revealed the difference in joints kinematics during different phases of the running stance, the ankle joint exhibited larger dorsiflexion and plantarflexion in the non-dominant limb, which is the left leg, while the dominant limb exhibited larger flexion in both knee joint and hip joint. The joint angle velocity also showed different significant differences in all three lower extremity joints. Theoretically, asymmetrical gait has the potential to abnormally or injuriously load the lower extremity [24]. Previous studies have reported significant gait asymmetry between the injured and uninjured limbs among runners with a history of unilateral overuse injury [28], [29]. Gilgen-Ammann et al. [14] concluded that a previous injury was associated with bilateral asymmetry, as the runners who had a previous injury having significantly enlarged bilateral asymmetry compared to those without injury history. However, we did not observe that a particular limb side of joint kinematic variables consistently scored higher, which may contrast with Gundersen et al.'s [16] findings, while there are some asymmetries existing in the lower limbs, they could not be correlated with lower extremity laterality. As kinematic waveforms of lower limb joints and segments were dissimilar between runners with different running volumes [4]. Carpes et al. [8] found that well-trained subjects showed a lower level of bilateral asymmetry in exercise and better perception of bilateral asymmetry compared to subjects who had just started a training regimen. Bilateral asymmetry was found to be related to running experience. The significant asymmetry between the lower limbs may be due to novice runners lack of running 
experience. This is in contrast with experienced runners who are defined as runners who have been participating in running competitions, and running regularly for more than 4 years and had a minimum weekly running distance of $30-80 \mathrm{~km}$ [20]. These findings may have immediate implications for the treatment of running-related injuries.

A previous study suggested imbalances of propulsion and maximal downward vertical displacement measures increase with increases in speed [2], indicating a potential for greater gait asymmetry in running. However, while comparing the bilateral asymmetry changes of the lower limbs during different running speeds, Girard et al. [15] observed no significant difference in group mean and the range of gait bilateral asymmetry values across different running speeds. Mo et al. [19] found changes of gait bilateral asymmetry across speeds were inconsistent and dependent on parameters of interest among novice runners. In the current study, except for one variable (i.e., peak ankle plantarflexion between the running speed of $10 \mathrm{~km} / \mathrm{h}$ and $12 \mathrm{~km} / \mathrm{h}$ ), we found no differences in the symmetry angle (SA) scores across different running speeds. Even for joint angle velocities, for which the average values shift drastically, the level of gait asymmetry was consistently small across all speeds. Minimal differences between lower limbs during running for different speeds suggest that the legs may not be used preferentially for braking or propulsion [15]. The main observation of our study is that left and right asymmetry values of running joints kinematics did not change as velocity varied between 8 and $12 \mathrm{~km} / \mathrm{h}$, which may indicate that the neuromuscular modulation of overall running speeds from 8 to $12 \mathrm{~km} / \mathrm{h}$ state, SA becomes more consistent.

The data presented in this study are novel as we applied SPM to detect gait bilateral asymmetry in joints kinematic parameters between the left and right leg. However, there are some limitations. First, all running tests were completed on a treadmill for obtaining continuous kinematic data. Although running kinematics were similar between treadmill and overground running, gait bilateral asymmetries were reported differently between the two conditions [25]. Second, we only assessed sagittal plane joint kinematics during the running stance phase, as bilateral asymmetries may have also affected frontal plane kinematics, and bilateral asymmetries of the running swing phase may be different between lower limbs. Third, fixed running speeds from 8 to $12 \mathrm{~km} / \mathrm{h}$ were employed for all runners regardless of their preferred running speed, as it is reported preferred running speed exhibited lower bilateral asymmetry than non- preferred running speeds [12]. Last, the subjects of this study were all male novice runners. As competitive level and gender have influences on the joint kinematics during running, the results might be not the same for other populations, which needs to be considered in future studies.

\section{Conclusion}

In summary, gait asymmetry of joint kinematics variables between lower limbs during running is apparent in healthy novice runners. The results of the current study also demonstrated during fresh-state (non-fatigued) treadmill running, bilateral asymmetry is largely unaffected by increasing running speed from 8 to $12 \mathrm{~km} / \mathrm{h}$. These data indicate that different running speeds have no meaningful effects on the sensitivity of detecting gait asymmetries among non-injured, novice runners. The findings provide implications for potential running-related injury risk, future research is needed to investigate asymmetry and lower extremity injury development.

\section{References}

[1] BAltich J., EMERY C., WhitTAKer J., NigG B., Running injuries in novice runners enrolled in different training interventions: a pilot randomized controlled trial, Scandinavian Journal of Medicine and Science in Sports, 2017, 27 (11), 1372-1383, DOI: 10.1111/sms.12743.

[2] Zhang B., Lu Q., A Current Review of Foot Disorder and Plantar Pressure Alternation in the Elderly, Physical Activity and Health, 2020, 4 (1), 95-106, DOI: 10.5334/paah.57.

[3] Bezodis I.N., Kerwin D.G., Salo A., Lower-limb mechanics during the support phase of maximum-velocity sprint running, Medicine and Science in Sports and Exercise, 2008, 40 (4), 707-715, DOI: 10.1249/MSS.0b013e318162d162.

[4] BOYer K.A., Silvernail J.F., HAMiLl J., The role of running mileage on coordination patterns in running, Journal of Applied Biomechanics, 2014, 30 (5), 649-654. 30, 649-654, DOI: 10.1123/JAB.2013-0261.

[5] Bredeweg S., Buist I., Kluitenberg B., Differences in kinetic asymmetry between injured and noninjured novice runners: a prospective cohort study, Gait \& Posture, 2013, 38 (4), 847-852, DOI: 10.1016/j.gaitpost.2013.04.014.

[6] Buist I., Bredeweg S.W., Bessem B., Van Mechelen W., LEMMINK K.A., DIERCKS R.L., Incidence and risk factors of running-related injuries during preparation for a 4-mile recreational running event, British Journal of Sports Medicine, 2010, 44 (8), 598-604, DOI: 10.1136/bjsm.2007.044677.

[7] Buist I., Bredeweg S.W., Lemmink K.A., Van Mechelen W., DIERCKS R.L., Predictors of running-related injuries in novice runners enrolled in a systematic training program: a prospective cohort study, The American Journal of Sports Medicine, 2010, 38 (2), 273-280, DOI: 10.1177/0363546509347985. 
[8] CARPes F.P., Bini R.R., Mota C.B., Training level, perception and bilateral asymmetry during multijoint leg-press exercise, Brazilian Journal of Biomotricity, 2008, 2 (2), 51-62.

[9] Ferber R., Davis I.M., Williams III D.S., Gender differences in lower extremity mechanics during running, Clinical Biomechanics, 2003, 18(4), 350-357, DOI: 10.1016/ S0268-0033(03)00025-1.

[10] Foch E., Reinbolt J.A., Zhang S., Fitzhugh E.C., Milner C.E., Associations between iliotibial band injury status and running biomechanics in women, Gait \& Posture, 2015, 41 (2), 706-710, DOI: 10.1016/j.gaitpost.2015.01.031.

[11] FuKuChI R.K., FuKuchi C.A., DuARTE M., A public dataset of running biomechanics and the effects of running speed on lower extremity kinematics and kinetics, PeerJ., 2017, 5, e3298, DOI: $10.7717 /$ peerj.3298.

[12] Furlong L.-A., EgGinton N.L., Kinetic asymmetry during running at preferred and non-preferred speeds, Medicine and Science in Sports and Exercise, 2018, 50 (6), 1241-1248, DOI: $10.1249 / \mathrm{MSS} .0000000000001560$.

[13] Gao Z., Mei Q., Fekete G., BaKer J.S., Gu Y., The Effect of Prolonged Running on the Symmetry of Biomechanical Variables of the Lower Limb Joints, Symmetry, 2020, 12 (5), 720, DOI: $10.3390 /$ sym 12050720 .

[14] Gilgen-Ammann R., Taube W., Wyss T., Gait asymmetry during 400-to 1000-m high-intensity track running in relation to injury history, International Journal of Sports Physiology and Performance, 2017, 12 (s2), S2-157-S2-60, DOI: 10.1123/ijspp.2016-0379.

[15] Girard O., Morin J.-B., Ryu J., Read P., Townsend N., Running velocity does not influence lower limb mechanical asymmetry, Frontiers in Sports and Active Living, 2019, 1 (36), DOI: $10.3389 /$ fspor.2019.00036.

[16] Gundersen L.A., Valle D.R., Barr A.E., Danoff J.V., STANHOPE S.J., SNYDER-MACKLER L., Bilateral analysis of the knee and ankle during gait: an examination of the relationship between lateral dominance and symmetry, Physical Therapy, 1989, 69 (8), 640-650, DOI: 10.1093/ptj/69.8.640.

[17] JiANG C., The Effect of Basketball Shoe Collar on Ankle Stability: A Systematic Review and Meta-Analysis, Physical Activity and Health, 2020, 4 (1), 11-18, DOI: 10.5334/paah.48.

[18] Lun V., Meeuwisse W., Stergiou P., Stefanyshyn D., Relation between running injury and static lower limb alignment in recreational runners, British Journal of Sports Medicine, 2004, 38 (5), 576-580, DOI: 10.1136/bjsm.2003.005488.

[19] Mo S., LAU F.O., LOK A.K., CHAN Z.Y., ZhaNG J.H., SHUM G., CHEUNG R.T., Bilateral asymmetry of running gait in competitive, recreational and novice runners at different speeds, Human Movement Science, 2020, 71, 102600, DOI: 10.1016/ j.humov.2020.102600.
[20] Mo S., CHow D.H.K., Differences in lower-limb coordination and coordination variability between novice and experienced runners during a prolonged treadmill run at anaerobic threshold speed, Journal of Sports Sciences, 2018, 37, 1021-1028, DOI: 10.1080/02640414.2018.1539294.

[21] Pairot De Fontenay B., Argaud S., Blache Y., Monteil K., Asymmetries in joint work during multi-joint movement after anterior cruciate ligament reconstruction: A pilot study, Scandinavian Journal of Medicine \& Science in Sports, 2014, 24 (6), e471-476, DOI: 10.1111/sms.12207.

[22] PATAKy T.C., One-dimensional statistical parametric mapping in Python, Computer Methods in Biomechanics and Biomedical Engineering, 2012, 15 (3), 295-301, DOI: 10.1080/ 10255842.2010.527837.

[23] Quan W., Wang M., Liu G., Fekete G., Baker J.S., Ren F., Gu Y., Comparative Analysis of Lower Limb Kinematics between the Initial and Terminal Phase of $5 \mathrm{~km}$ Treadmill Running, Journal of Visualized Experiments: Jove, 2020, (161), DOI: 10.3791/61192.

[24] RadZak K.N., Putnam A.M., Tamura K., Hetzler R.K., STICKLEY C.D., Asymmetry between lower limbs during rested and fatigued state running gait in healthy individuals, Gait and Posture, 2017, 51, 268-274, DOI: 10.1016/ j.gaitpost.2016.11.005.

[25] Robadey J., Staudenmann D., Schween R., Gehring D., Gollhofer A., TAUBE W., Lower between-limb asymmetry during running on treadmill compared to overground in subjects with laterally pronounced knee osteoarthritis, Plos one, 2018, 13 (10), e0205191, DOI: 10.1371/journal.pone.0205191.

[26] SADEGHI H., Local or global asymmetry in gait of people without impairments, Gait and Posture, 2003, 17 (3), 197-204, DOI: 10.1016/S0966-6362(02)00089-9.

[27] Sadeghi H., Allard P., Prince F., Labelle H., Symmetry and limb dominance in able-bodied gait: a review, Gait and Posture, 2000, 12 (1), 34-45, DOI: 10.1016/ S0966-6362(00)00070-9.

[28] ZiFCHOCK R.A., DAvis I., HAMILL J., Kinetic asymmetry in female runners with and without retrospective tibial stress fractures, Journal of Biomechanics, 2006, 39 (15), 2792-2797, DOI: 10.1016/j.jbiomech.2005.10.003.

[29] ZifChOCK R.A., Davis I., Higginson J., MCCAW S., ROYER T., Side-to-side differences in overuse running injury susceptibility: a retrospective study, Human Movement Science, 2008, 27 (6), 888-902, DOI: 10.1016/ j.humov.2008.03.007.

[30] ZifChock R.A., Davis I., Higginson J., Royer T., The symmetry angle: a novel, robust method of quantifying asymmetry, Gait and Posture, 2008, 27 (4), 622-627, DOI: 10.1016/ j.gaitpost.2007.08.006. 